\title{
Feature Extraction based on Generalized \\ Permutation Entropy for Condition Monitoring of Rotating Machinery
}

Jinshan Lin ( $\nabla$ jslinmec@hotmail.com )

Weifang University https://orcid.org/0000-0002-3163-9873

Chunhong Dou

Weifang University

Yingjie Liu

Weifang University

\section{Research Article}

Keywords: Feature extraction, permutation entropy, multiscale permutation entropy, general-ized permutation entropy, condition monitoring, rotating machinery

Posted Date: July 16th, 2021

DOI: https://doi.org/10.21203/rs.3.rs-680265/v1

License: (a) (1) This work is licensed under a Creative Commons Attribution 4.0 International License. Read Full License

Version of Record: A version of this preprint was published at Nonlinear Dynamics on November 22nd, 2021. See the published version at https://doi.org/10.1007/s11071-021-07054-2. 


\title{
Feature extraction based on generalized per- mutation entropy for condition monitoring of rotating machinery
}

\author{
Jinshan Lin ${ }^{\mathrm{a}, *}$, Chunhong Dou ${ }^{\mathrm{b}}$, Yingjie Liu ${ }^{\mathrm{a}}$ \\ ${ }^{a}$ School of Mechatronics and Vehicle Engineering, Weifang University, Weifang \\ 261061, China \\ ${ }^{b}$ School of Information and Control Engineering, Weifang University, Weifang \\ 261061, China \\ *Corresponding author: jslinmec@ @otmail.com, jslinmec@wfu.edu.cn (J. Lin), Tel \& Fax: +86 \\ 5368785603
}

\begin{abstract}
Defective rotating machinery usually exhibits complex dynamic behavior. Therefore, feature representation of machinery vibration signals is always critical for condition monitoring of rotating machinery. Permutation entropy (PeEn), an adaptive symbolic description, can measure complexities of signals. However, PeEn may lack the capability to fully describe dynamics of complex signals since compressing all the information into a single parameter. Afterwards, multiscale PeEn (MPeEn) is put forward for coping with nonstationarity, outliers and artifacts emerging in complex signals. In MPeEn, a set of parameters serve to describe dynamics of a complex signal in different time scales. Nonetheless, an average procedure in MPeEn may withhold local information of a complex signal. To overcome deficiencies of PeEn and MPeEn, this paper proposes generalized PeEn (GPeEn) by introducing different time lags and orders into PeEn. In GPeEn, a complex signal is compressed into one matrix rather than a single parameter. Moreover, minimal, maximal and average values of a matrix obtained by GPeEn serve to briefly describe conditions of rotating machinery. Next, a numerical experiment proves that GPeEn outperforms PeEn and MPeEn in characterizing conditions of a Lorenz model. Subsequently, the performance of GPeEn is benchmarked against that of PeEn and MPeEn by investigating gear and roll-bearing vibration signals containing different types and severity of faults. The results show that the proposed method has a clear advantage over PeEn and MPeEn in condition monitoring of rotating machinery.
\end{abstract}

Keywords: Feature extraction, permutation entropy, multiscale permutation entropy, generalized permutation entropy, condition monitoring, rotating machinery

\section{Introduction}

Defective rotating machinery usually displays complex nonstationarity and nonlinearity[1-3]. As a result, feature representation of machinery vibration signals is always a central problem for condition monitoring of rotating machinery[2-4]. Currently, some methods for time-frequency analysis, such as wavelet transform 
(WT) and empirical mode decomposition (EMD), have been exploited for feature extraction of machinery vibration signals[5-8]. However, either of WT and EMD seemingly encounters some difficulties in analyzing complex machinery vibration signals[9-12]. A lot of references have indicated that vibration signals from defective rotating machinery display obvious fractal and chaotic properties[1,13-17]. Consequently, extraction of nonlinear features from vibration signals is critically important for gaining an insight into dynamics of machinery. Then, phase space reconstruction (PSR) has been adopted to reveal nature of complex machinery vibration signals[18,19]. Nevertheless, it seems difficult for PSR to determine two important parameters, i.e. the embedding dimension and the time delay[20]. Next, mutifractal detrended fluctuation analysis (MFDFA) was applied to examine dynamics of complex machinery vibration signals[1,14,16,17]. Nonetheless, MFDFA needs refining further since suffering from some shortages[1,17]. Hence, extraction of nonlinear features for condition monitoring of rotating machinery leaves much to be desired[21].

Symbolic dynamics, a coarse-graining or description-reduction method, can remain chaotic properties of complex signals but discards considerable details[22]. Therefore, a symbolic description is effective for demonstrating nature of complex signals[13,15]. Currently, symbolic dynamics has been employed to capture essence of machinery vibration signals $[13,15]$. Traditionally, a symbolic description converts an original signal into several symbols in terms of a preset threshold[22]. As a result, the threshold-based symbolic method is rather short of adaptation. Next, permutation entropy (PeEn), an adaptive symbolic description, was proposed for measuring complexities of signals[23]. After presetting a permutation order and a time lag, PeEn can adaptively transform an original signal into a set of symbols by comparing neighboring values of a signal. Currently, PeEn has found its application in various research fields[24-26]. However, PeEn may lack the capability to fully describe dynamics of complex signals since compressing all the information into a single parameter[27]. Afterwards, multiscale PeEn (MPeEn) was put forward for coping with nonstationarity, outliers and artifacts emerging in complex signals[28]. In MPeEn, a set of parameters serve to describe dynamics of complex signals in different time scales. Nonetheless, an average procedure in MPeEn may withhold local information of complex signals. In fact, a complex dynamic system may display different dynamic behavior in 
different permutation orders and time lags[29]. To relieve shortages of PeEn and MPeEn, this paper broads the definition of PeEn by introducing different permutation orders and time lags into PeEn. Thus, this paper proposes generalized PeEn (GPeEn) for characterizing dynamics of complex signals. Next, the performance of GPeEn was benchmarked against that of PeEn and MPeEn by analyzing time series generated in a Lorenz model. In addition, GPeEn was compared with PeEn and MPeEn by investigating gear and rolling-bearing vibration signal containing different types and severity of faults. The results show that GPeEn is clearly superior to PeEn and MPeEn in condition monitoring of rotating machinery.

This paper is structured as follows. Section II formulates PeEn and MPeEn, proposes GPeEn and develops feature extraction based on GPeEn. In Section III, the performance of the proposed method is benchmarked numerically against that of PeEn and MPeEn by examining a Lorenz model. In Section IV, the proposed method is compared experimentally with PeEn and MPeEn by investigating gear and bearing vibration signals containing different types and severity of faults. Finally, Section V concludes this paper.

\section{Generalized Permutation Entropy (GPeEn)}

\subsection{PeEn}

For a time series $x_{t}(t=1,2, \mathrm{~L}, T)$, there are $n$ ! possible permutations $\psi$ for order $n$. The relative frequency $p(\psi)$ for each permutation $\psi$ is given as follows.

$$
p(\psi)=\frac{F\left\{t \mid t \leq T-n,\left(x_{t+1}, \mathrm{~L}, x_{t+n}\right) \text { has type } \psi\right\}}{T-n+1}
$$

The permutation entropy $h_{n}$ for order $n \geq 2$ is defined as follows.

$$
h_{n}=-\frac{\sum p(\psi) \log [p(\psi)]}{n-1}
$$

\subsection{GPeEn}

In GPeEn, different orders and time lags are considered. Suppose that GPeEn has lag size $L$ and order size $N$. Also, $\psi_{l, n}$ represents a permutation with lag $l$ 
$(1 \leq l \leq L)$ and order $n(1 \leq n \leq N)$. As such, the relative frequency $p\left(\psi_{l, n}\right)$ for each permutation $\psi_{l, n}$ is given as follows.

$$
p\left(\psi_{l, n}\right)=\frac{F\left\{t \mid t \leq T-n l,\left(x_{t+l}, x_{t+2 l} \mathrm{~L}, x_{t+n l}\right) \text { has type } \psi_{l, n}\right\}}{T-n l+1}
$$

The permutation entropy $h_{l, n}$ for order $n \geq 2$ is defined as follows.

$$
h_{l, n}=-\frac{\sum p\left(\psi_{l, n}\right) \log \left[p\left(\psi_{l, n}\right)\right]}{n-1}
$$

As given in Eq. (4), GPeEn, as opposed to PeEn, converts a time series to a matrix whose sizes are $L \times N$.

\subsection{Feature extraction based on GPeEn}

GPeEn seems largely redundant in describing properties of complex signals. Afterwards, this paper exploits minimal, maximal and average values of GPeEn to briefly describe dynamics of complex signals. Consequently, this paper proposes a novel method for feature extraction based on GPeEn.

\section{Application of GPeEn to Lorenz chaotic series}

The feasibility of the proposed method was evaluated by examining time series generated in a Lorenz model, which is formulized as follows.

$$
\left\{\begin{array}{c}
\&=\sigma(y-x) \\
\&=r x-y-x z \\
\&=x y-b z
\end{array}\right.
$$

In the Lorenz model, $\sigma=10$ and $b=8 / 3$. As known well, the Lorenz model demonstrates different dynamics for different Rayleigh numbers $r$ [30]. When $0<r<13.9656$, the Lorenz model does not contain chaos[30]. When 13.9656 $<r<24.06$, the Lorenz model contains transient chaos[30]. When $r>24.06$, the Lorenz model contains chaos[30]. In this paper, $r$ takes a value at a time from [0.7 1.2 10192124.53040$]$. Then, the Lorenz model is solved using the 4-order Runge-Kutta algorithm. First, PeEn was applied to examine the solutions of $x$ for different $r$ and the results are demonstrated in Fig. 1. As demonstrated in Fig. 1, PeEn remains constant for $r=0.7,1.2$ and 10 and does the same for $r=24.5,30$ and 40. Although successful in distinguishing between non-chaos, transient chaos and chaos, PeEn fails to distinguish between conditions for $r=0.7$, 
1.2 and between those for $r=24.5,30$ and 40. In the following, MPeEn was employed to decode the solutions of $x$ for different $r$ and the results are demonstrated in Fig. 2. As demonstrated in Fig. 2, although capable of distinguishing chaos from the other conditions, MPeEn can not distinguish between non-chaos and transient chaos. Next, GPeEn was employed to analyze the solutions of $x$ for different $r$ and the results are shown in Fig. 3. As shown in Fig. 3, time lags and orders exert a considerable impact on PeEn of the solutions of $x$ in the Lorenz model. Thus, GPeEn greatly varies with changes of time lags and orders. As a result, non-chaos, transient chaos and chaos can be clearly identified by GPeEn. Afterwards, the feature extraction method proposed in this paper was used to extract features from matrixes acquired by GPeEn and the results are displayed in Fig. 4. As displayed in Fig. 4, the proposed method in this paper can exactly describe conditions of the Lorenz model. In addition, Fig.4 illustrates that the time lag and the order both in PeEn and in MPeEn are hard to choose since valid for all the conditions.

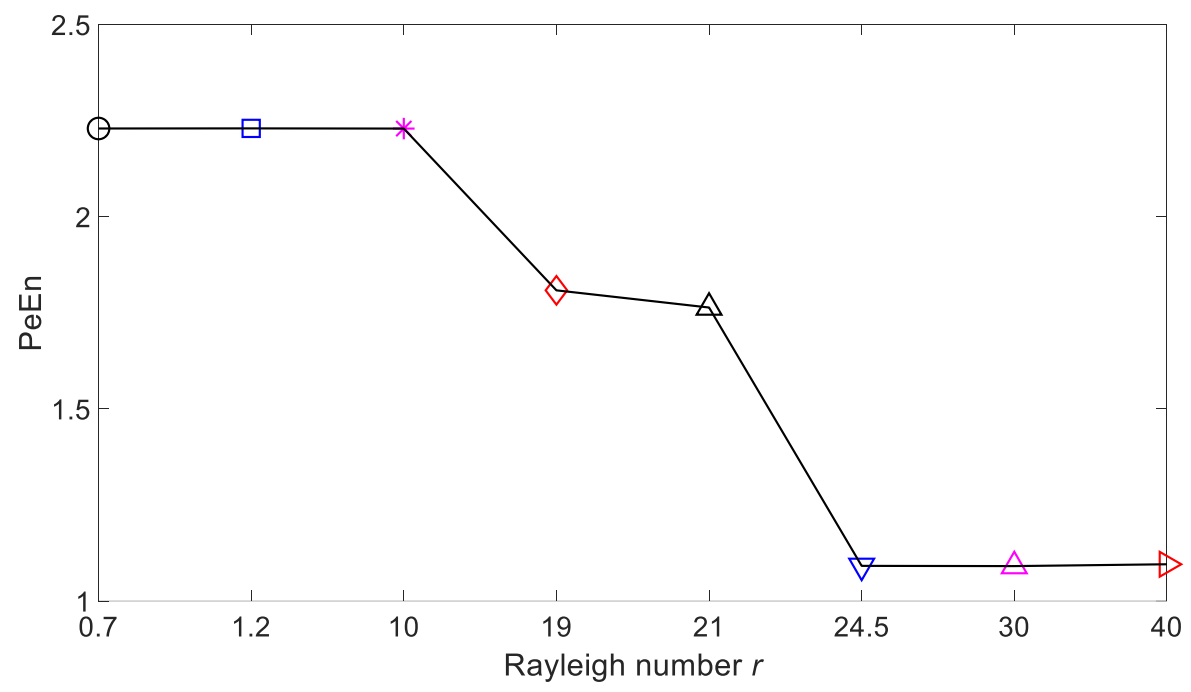

Fig. 1 PeEn of the solutions of $x$ in the Lorenz model for different Rayleigh numbers $r$. 


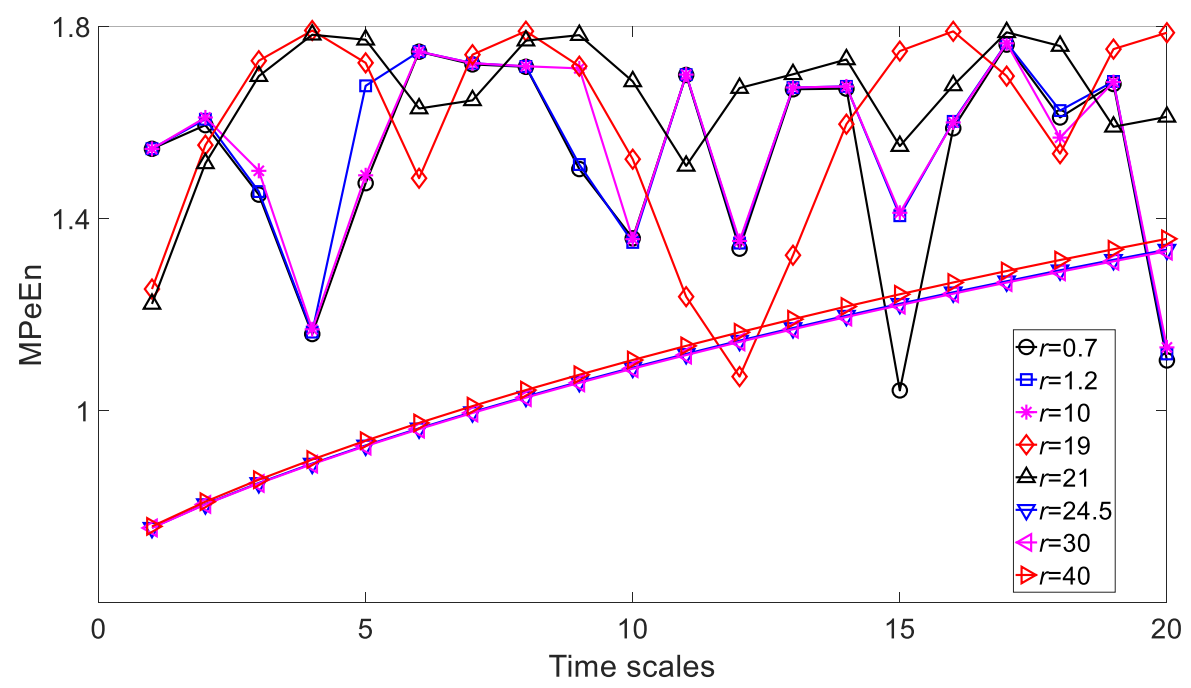

Fig. 2 MPeEn of the solutions of $x$ in the Lorenz model for different time scales.

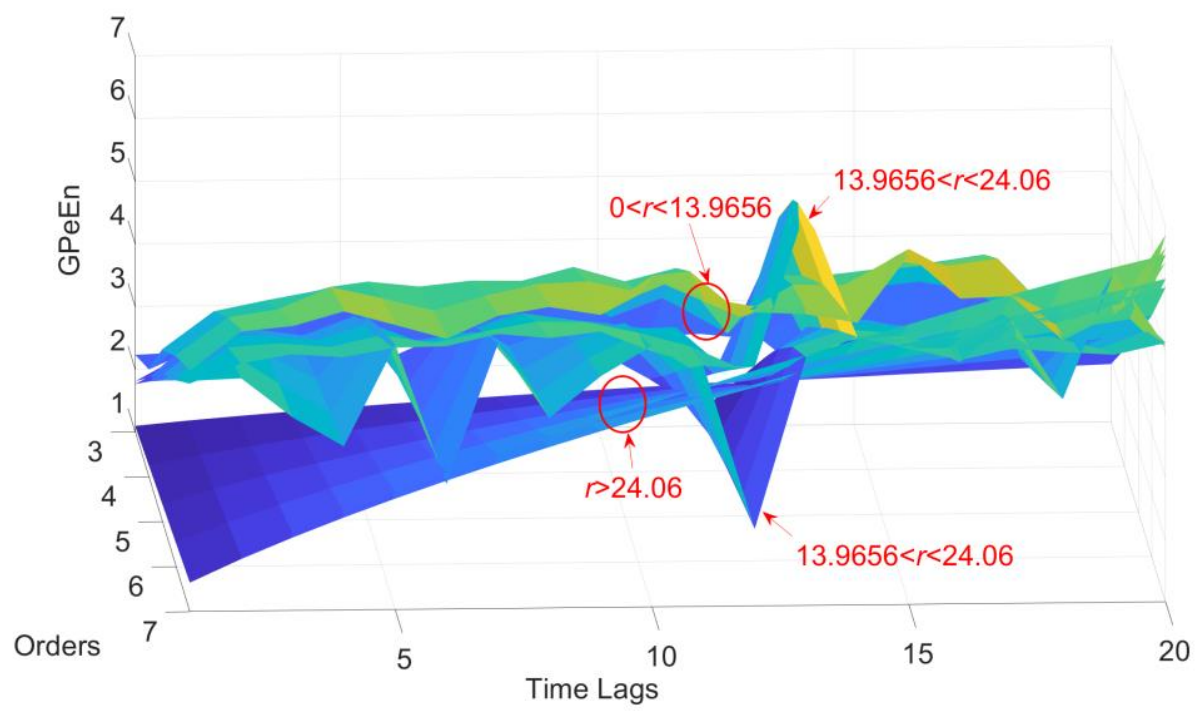

Fig. 3 GPeEn of the solutions of $x$ in the Lorenz model for $r=0.7,1.2,10,19,21,24.5,30,40$. 


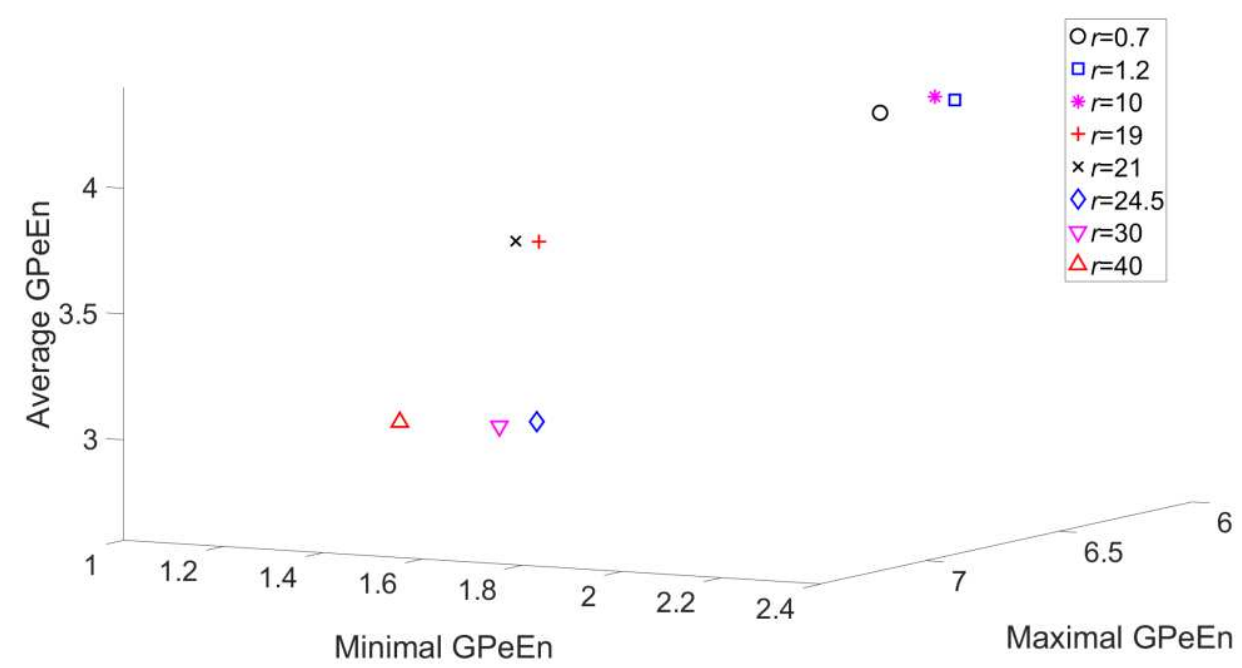

Fig. 4 Minimal, maximal and average values of GPeEn of the solutions of $x$ in the Lorenz model for $r=0.7,1.2,10,19,21,24.5,30,40$.

\section{Condition monitoring of rotating machinery}

\subsection{Condition monitoring of gears}

This subsection conducted a gear experiment for modelling gear faults. In the gear experiment described in Fig 5, a driving motor has a constant speed of 1600 revolutions per minute (RPM). The gear experiment comprises four different types of gear condition: normal, slight-scratch, severe-scratch and broken-tooth. Sixteen pieces of vibration signals with a sample frequency of $16384 \mathrm{~Hz}$ and a size of 10000 points were captured from the driving end for each gear condition in the gear experiment. These four types of gear vibration signal are demonstrated in Fig. 6.

To begin with, PeEn was applied to investigate these gear vibration signals and the results are demonstrated in Fig. 7. As demonstrated in Fig. 7, PeEn can serve to distinguish between these four types of gear condition. However, PeEn for severe-scratch and broken-tooth fluctuates considerably over times. Next, MPeEn was adopted to examine these gear vibration signals and the results are illustrated in Fig. 8. As illustrated in Fig. 8, MPeEn for these four types of gear condition overlap greatly. In addition, MPeEn for these four types of gear vibration signal fluctuates widely over time. Thus, MPeEn seemingly demonstrates little feasibility for discriminating between these four types of gear condition. In the following, GPeEn was made use of analyzing these gear vibration signals. 
Moreover, minimal, maximal and average values of GPeEn served to distinguish between these four types of gear condition and the results are indicated in Fig. 9. As indicated in Fig. 9, these four types of gear condition can be clearly separated by the proposed method in this paper.

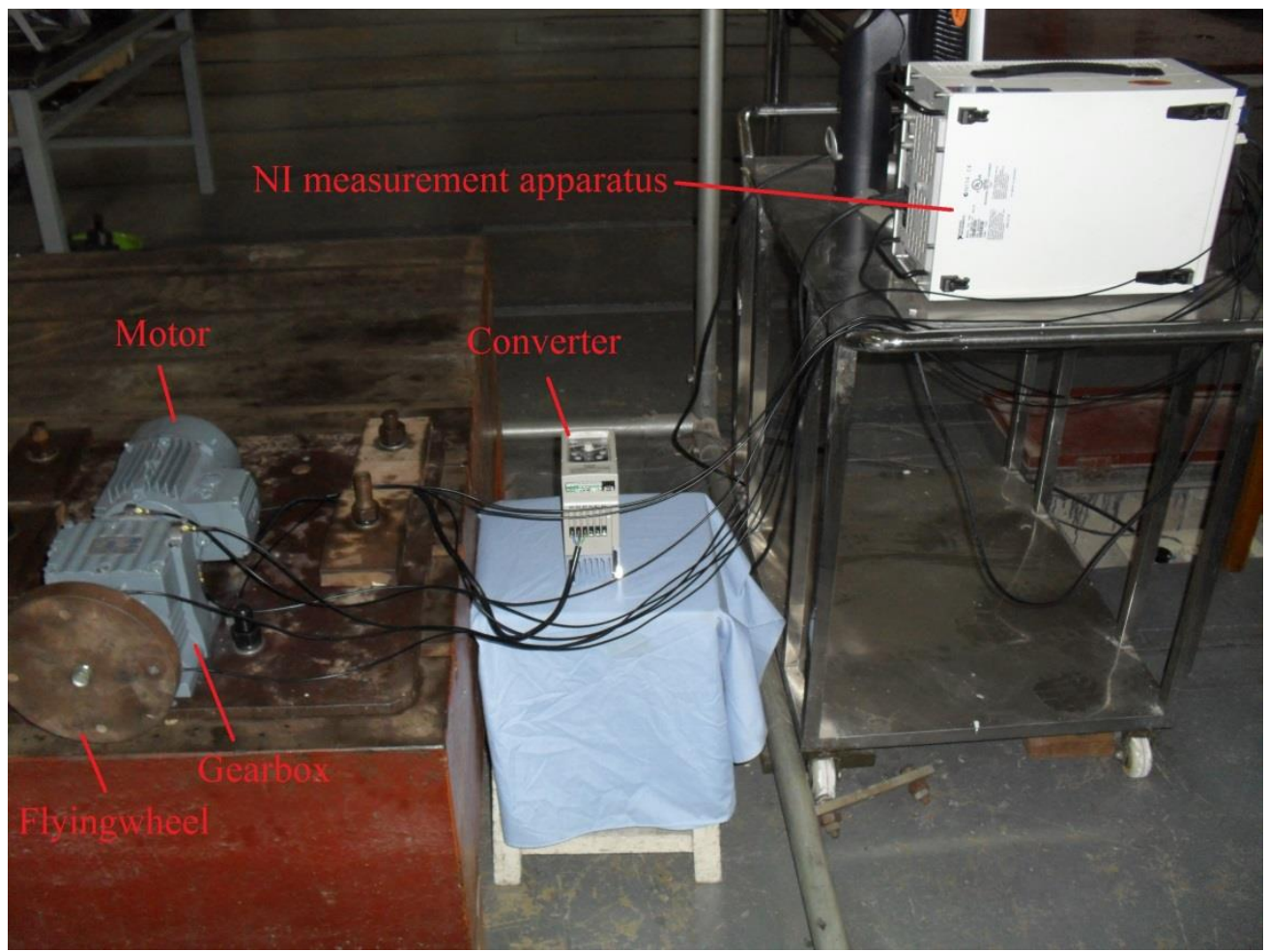

Fig. 5 A gear experiment for modelling gear faults.

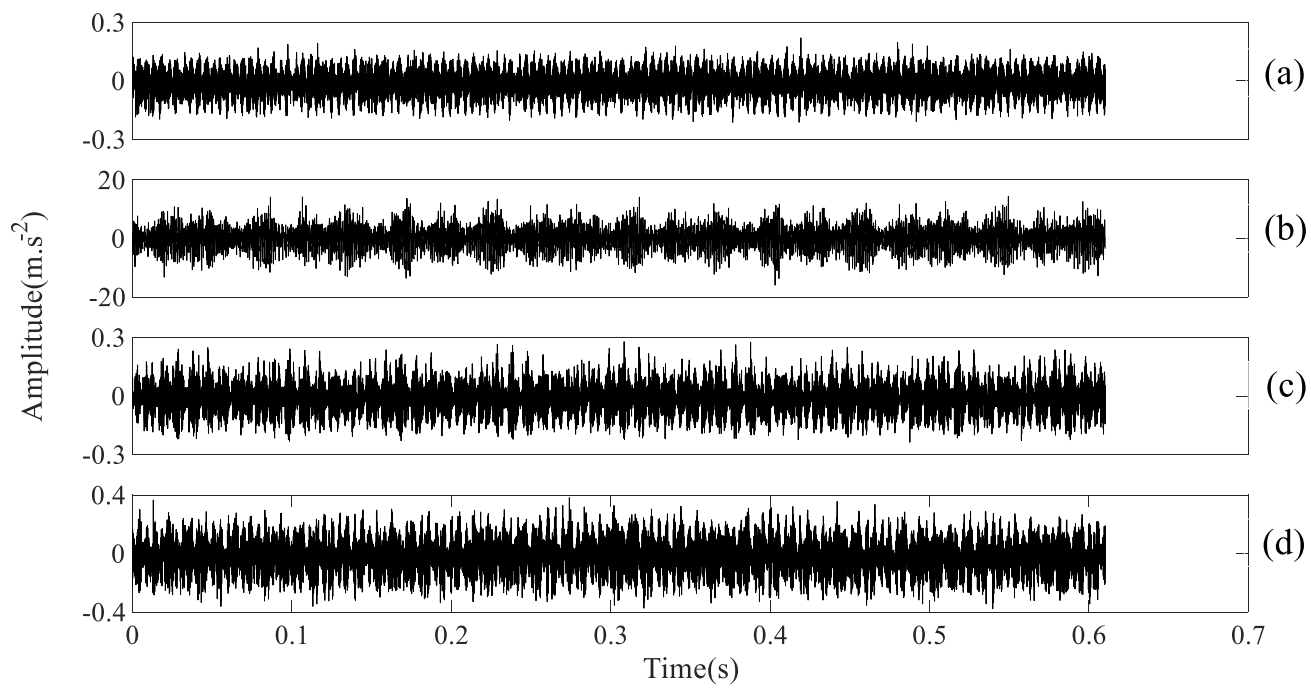

Fig. 6 Four types of gear vibration signal, (a) (d) for normal, slight-scratch, severe-scratch and broken-tooth, respectively. 


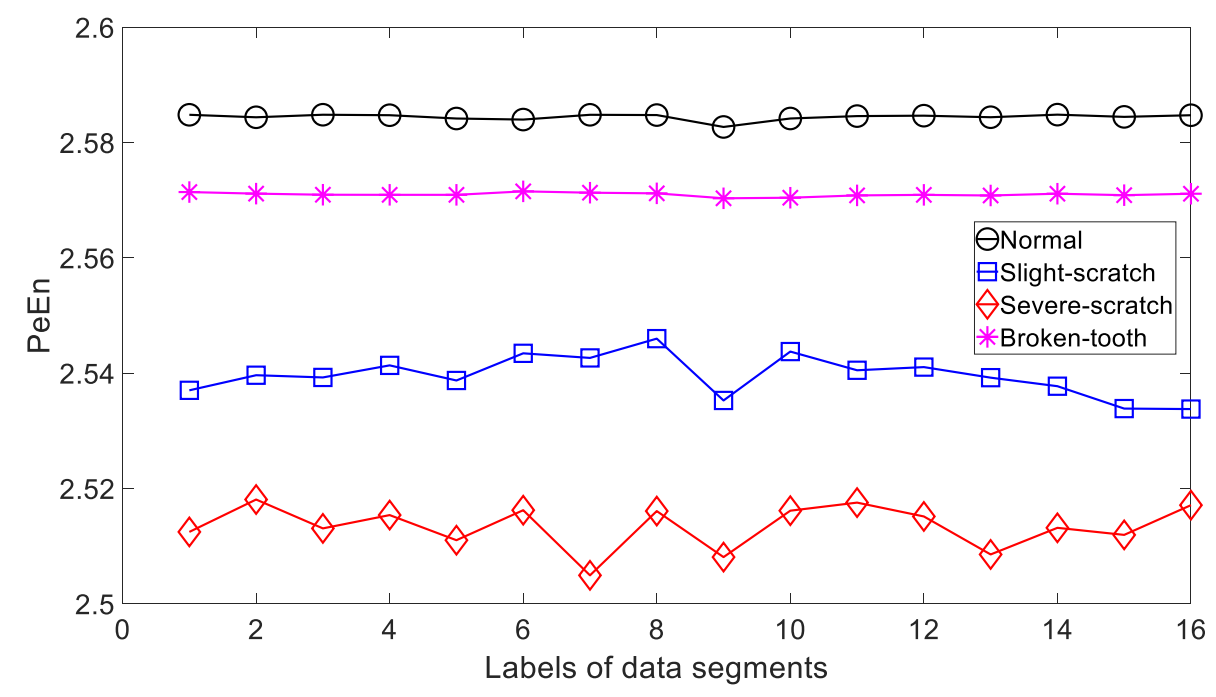

Fig. 7 PeEn for four types of gear vibration signal.

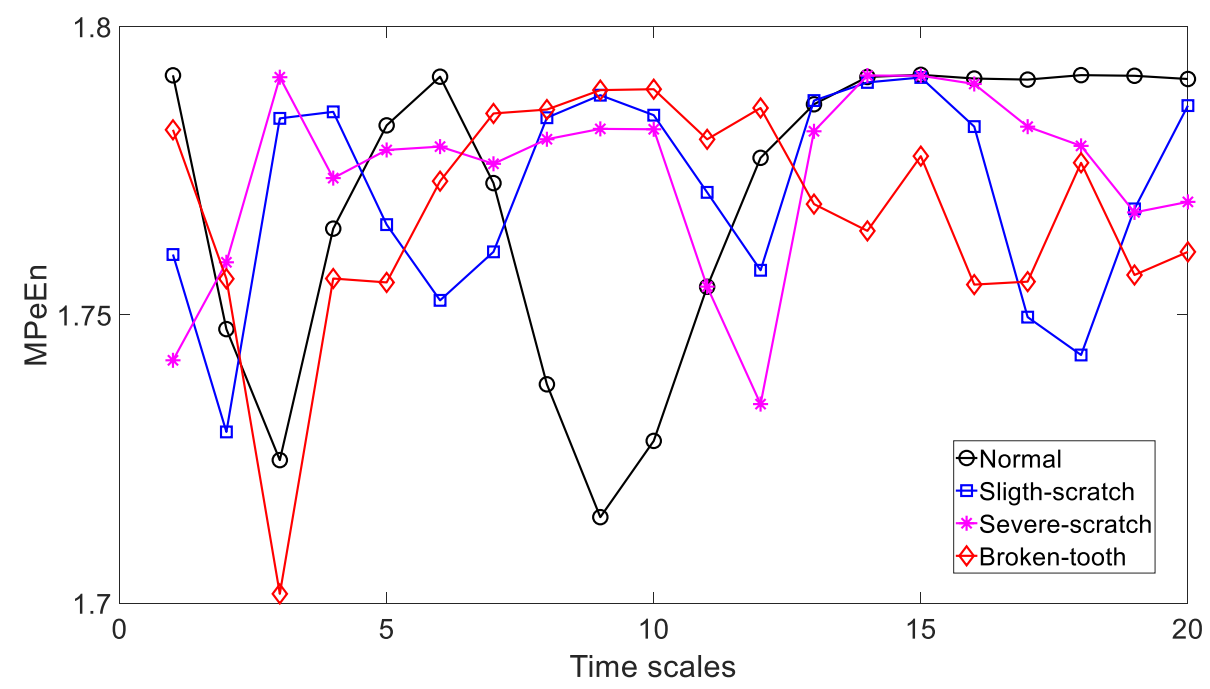

Fig. 8 MPeEn for four types of gear vibration signal.

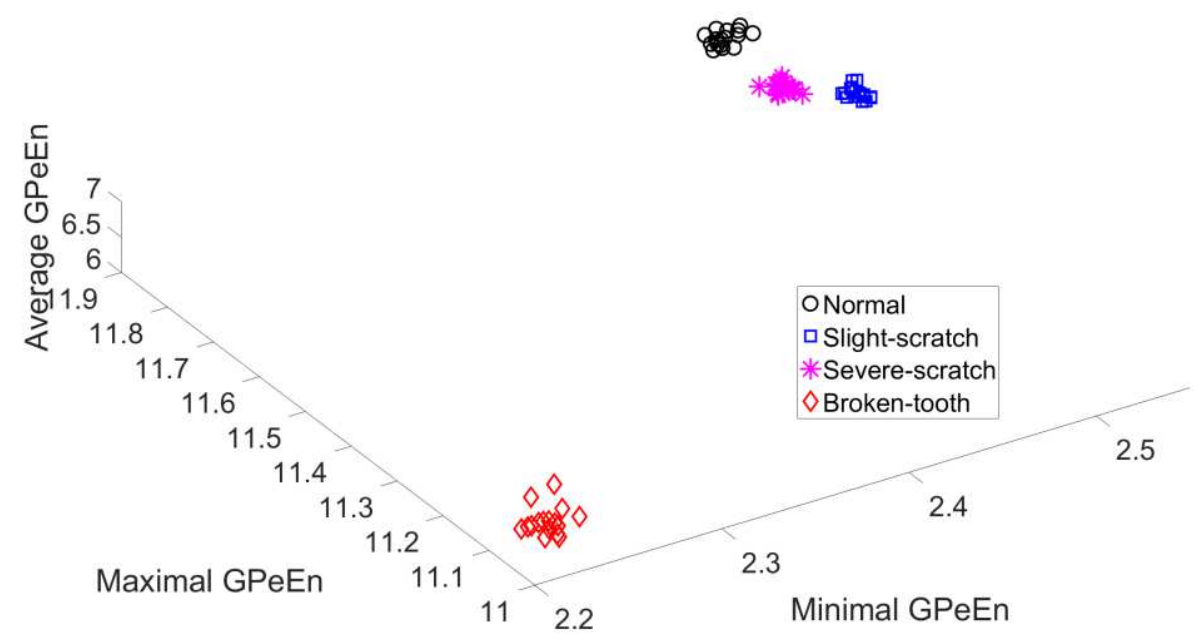

Fig. 9 Separation of four types of gear condition by the proposed method in this paper. 


\subsection{Condition monitoring of rolling bearings}

This subsection employed rolling-bearing vibration signals from Bearing Data Center of Case Western Reserve University to further measure the performance of the proposed method in this paper. In the bearing experiment described in Fig. 10, the revolving speed of a driving motor fluctuated between 1720 RPM and 1797 RPM. The bearing experiment included twelve different types of bearing condition: normal, an inner raceway fault with a fault diameter of 0.007 inches and a depth of 0.011 inches (IR007), an inner raceway fault with a fault diameter of 0.014 inches and a depth of 0.011 inches (IR014), an inner raceway fault with a fault diameter of 0.021 inches and a depth of 0.011 inches (IR021), an inner raceway fault with a fault diameter of 0.028 inches and a depth of 0.050 inches (IR028), a ball fault with a fault diameter of 0.007 inches and a depth of 0.011 inches (B007), a ball fault with a fault diameter of 0.014 inches and a depth of 0.011 inches (B014), a ball fault with a fault diameter of 0.021 inches and a depth of 0.011 inches (B021), a ball fault with a fault diameter of 0.028 inches and a depth of 0.150 inches (B028), an outer raceway fault with a fault diameter of 0.007 inches and a depth of 0.011 inches (OR007), an outer raceway fault with a fault diameter of 0.014 inches and a depth of 0.011 inches (OR014) and an outer raceway fault with a fault diameter of 0.021 inches and a depth of 0.011 inches (OR021). Six pieces of vibration signals with a sample frequency of $12000 \mathrm{~Hz}$ and a size of 20000 points were collected from the driving end for each bearing condition in the bearing experiment. These twelve types of bearing vibration signal are displayed in Fig. 11.

To start with, PeEn was utilized to explore these bearing vibration signals and the results are given in Fig. 12. As given in Fig. 12, except normal, IR028 and OR014, the remaining nine types of bearing condition are hard to separate. After this, MPeEn was used to study these bearing vibration signals and the results are depicted in Fig. 13. As depicted in Fig. 13, there is considerable overlap between MPeEn of these twelve types of bearing vibration signal. Furthermore, GPeEn was adopted to probe these bearing vibration signals and the results are illustrated in Fig. 14. As illustrated in Fig. 14, GPeEn has the capability to distinguish between these twelve types of bearing condition. 


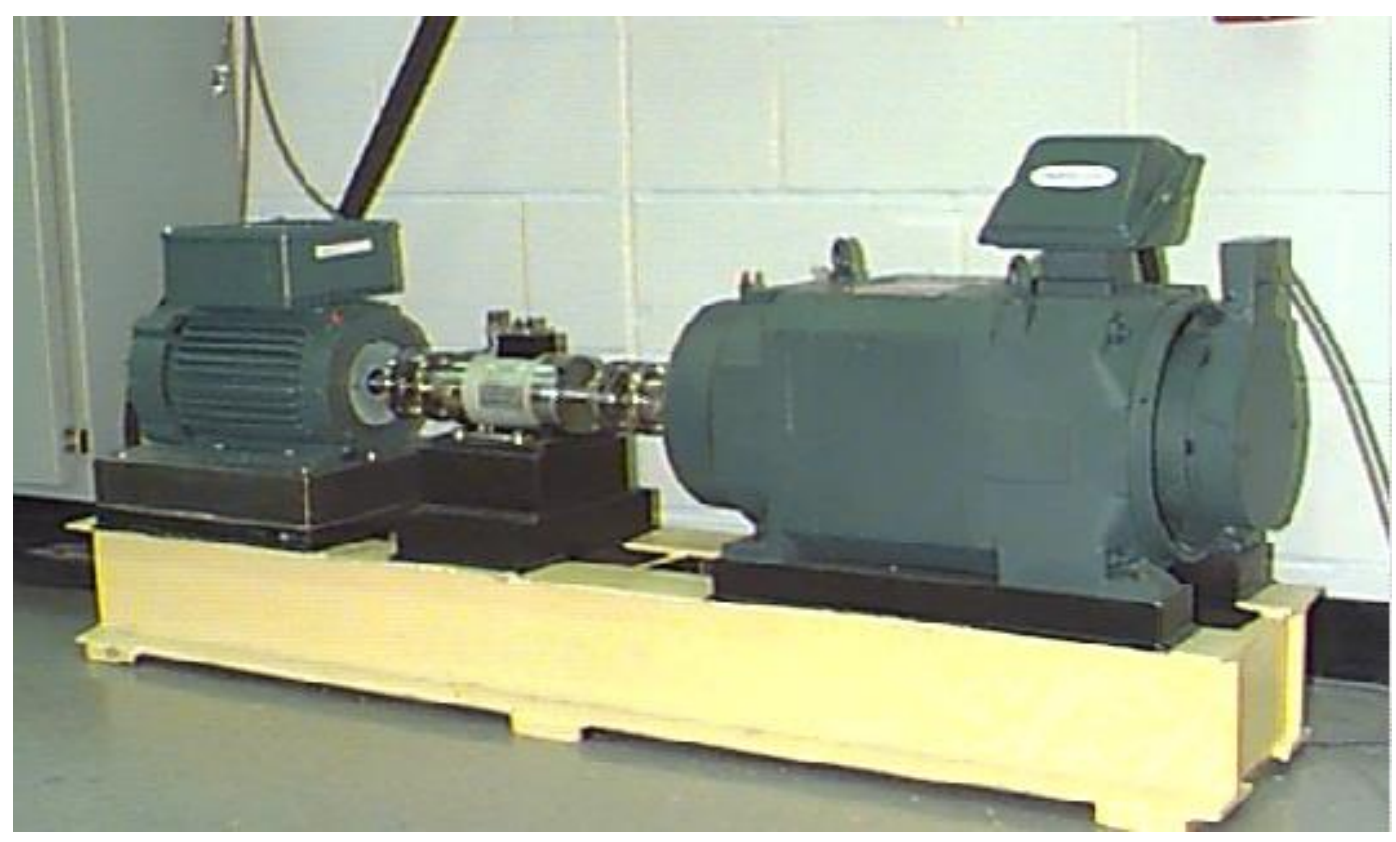

Fig. 10 A rolling-bearing experiment for modelling bearing faults.

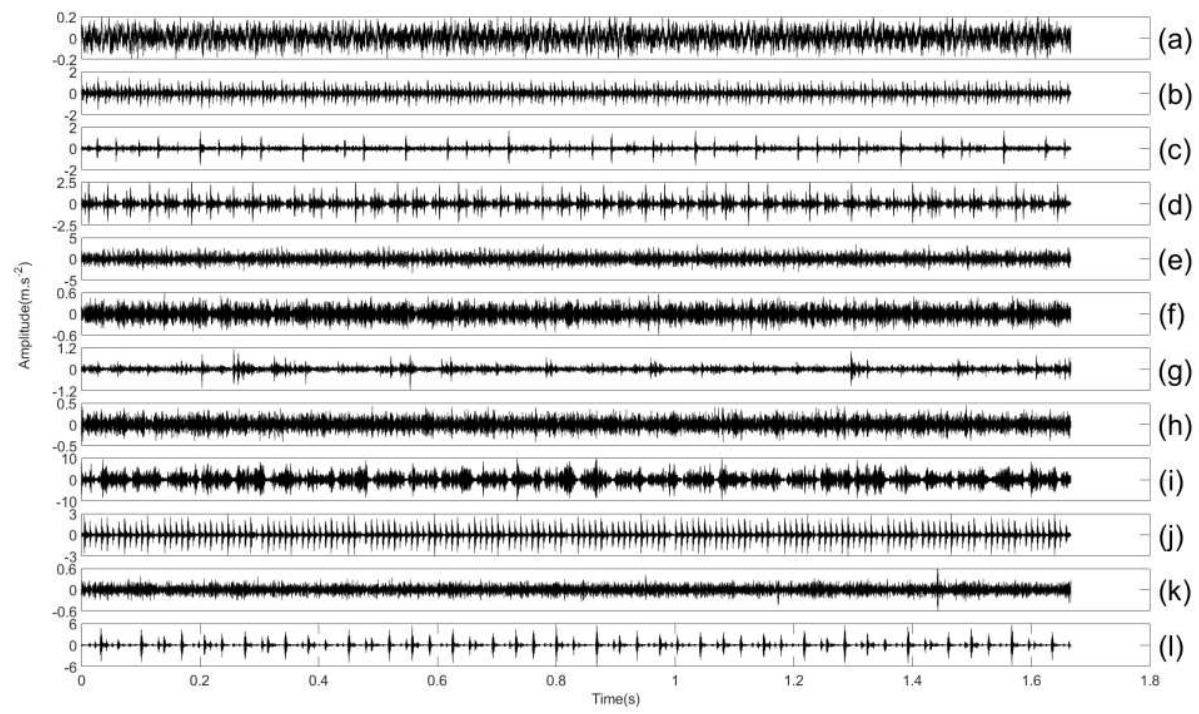

Fig. 11 Twelve types of bearing vibration signal in the rolling-bearing experiment, (a) (1) for normal, IR007, IR014, IR021, IR028, B007, B014, B021, B028, OR007, OR014 and OR021, respectively. 


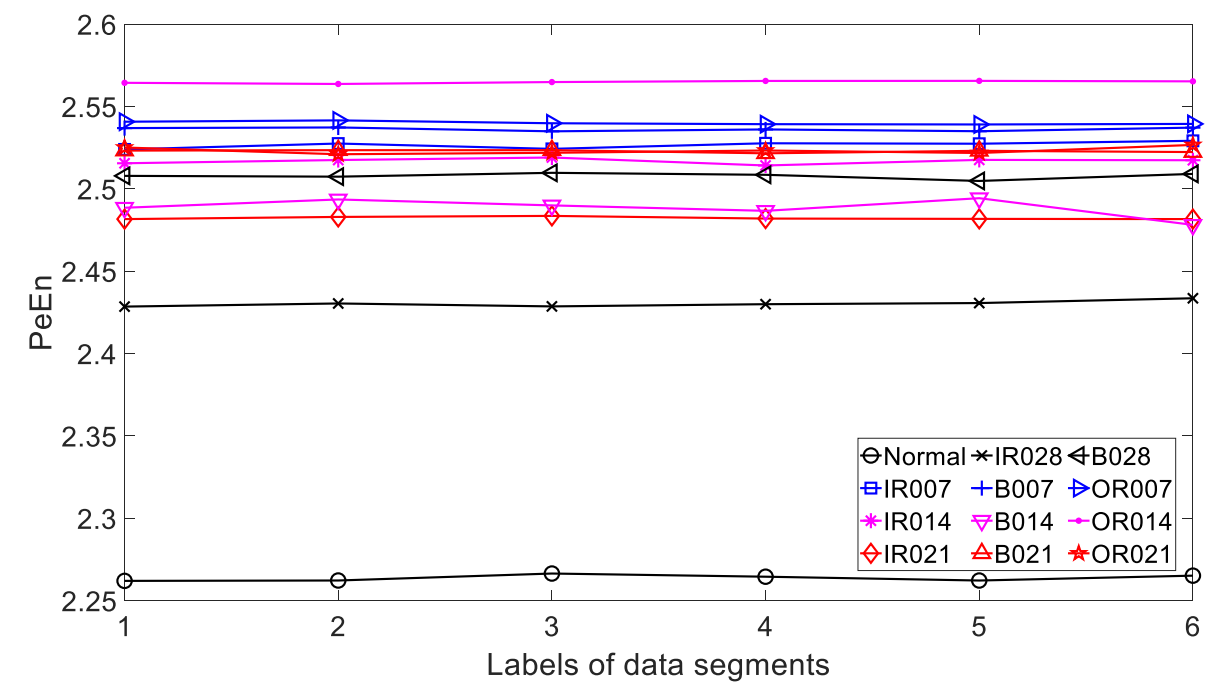

Fig. 12 PeEn for twelve types of bearing vibration signal.

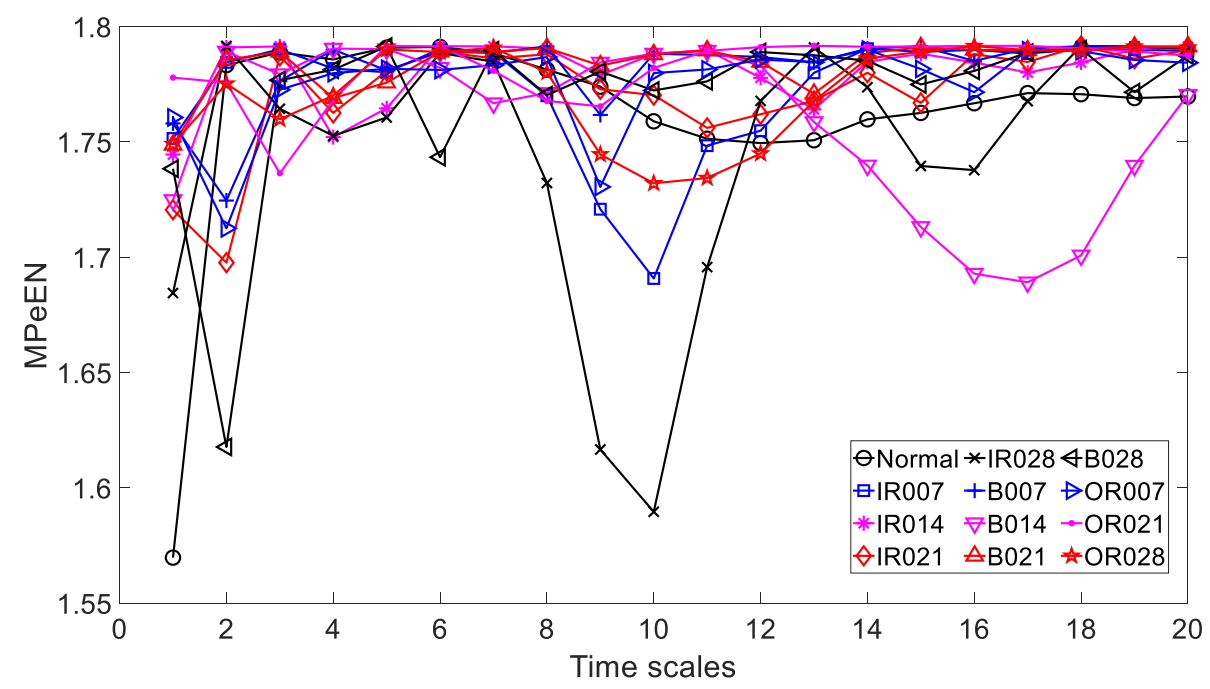

Fig. 13 MPeEn for twelve types of bearing vibration signal.

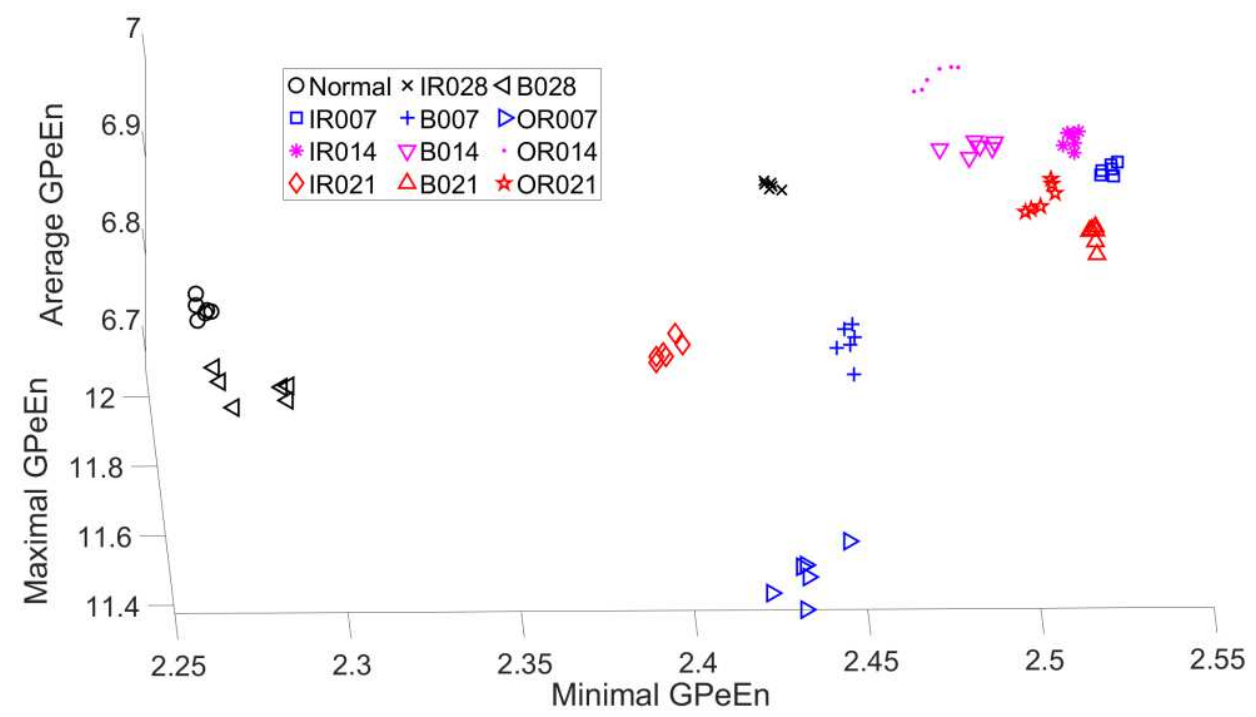

Fig. 14 Separation of twelve types of bearing condition by the proposed method in this paper. 


\subsection{Discussions}

This paper compares the performance of PeEn, MPeEn and GPeEn numerically and experimentally. Consequently, PeEn frequently undergoes a small change over time for a single machinery condition. This means that PeEn may not be highly reliable for characterizing conditions of machinery. In addition, MPeEn illustrates a considerable change over time for a single machinery condition due to an average procedure. This means that MPeEn may lack reliability for characterizing conditions of machinery. Thus, these comparisons show that GPeEn has a clear advantage over PeEn and MPeEn in describing conditions of machinery. Additionally, the proposed method in this paper delivers an excellent performance in distinguishing between different types and severity of machinery faults.

This paper makes two main contributions. First, this paper generalizes PeEn to GPeEn by introducing different time lags and orders into PeEn. Indeed, time lags and orders make a considerable impact on PeEn, as demonstrated in this paper. Accordingly, it is really necessary to consider time lags and orders for computing PeEn. As a consequence, GPeEn can more fully reflect nature of machinery vibration signals than PeEn. Second, minimal, maximal and average values of GPeEn are exploited to describe conditions of machinery. Consequently, this enables application of GPeEn in describing conditions of machinery. Both numerical and experimental examples prove that the proposed method in this paper can effectively characterize conditions of a complex dynamic system.

Although performing well in characterizing conditions of complex dynamic systems, the proposed method in this paper still run into some difficulties. For one thing, GPeEn has a higher time cost than PeEn. Thus, it seems slightly difficult for GPeEn as an online algorithm. For another, properties of a matrix obtained by GPeEn needs investigating further. These problems will be solved in the further.

\section{Conclusions}

This paper proposes GPeEn by introducing different time lags and orders into PeEn for relieving deficiencies of PeEn and MPeEn. Furthermore, a simulation proves that GPeEn performs better than PeEn and MPeEn in describing conditions of a Lorenz model. Afterwards, the performance of GPeEn was compared with that of PeEn and MPeEn by examining machinery vibration signals containing different types and severity of machinery faults. The results indicate that GPeEn 
delivers a better performance than PeEn and MPeEn in describing conditions of machinery. This paper seems to develop a powerful method for condition monitoring of rotating machinery.

\section{Acknowledgements}

Authors would like to kindly thank Bearing Data Center of Case Western Reserve University for sharing rolling-bearing fault data. The work was supported by Shandong Provincial Natural Science Foundation China (ZR2012EEL07).

\section{Data availability}

The datasets generated during and/or analysed during the current study are available from the corresponding author on reasonable request.

\section{Conflict of interest}

The authors declare that they have no conflict of interest.

\section{References}

1. Lin, J., Dou, C., Liu, Y.: Multifractal detrended fluctuation analysis based on optimized empirical mode decomposition for complex signal analysis. Nonlinear Dynam 103(3), 24612474 (2021). doi:10.1007/s11071-021-06223-7

2. Liu, Z., Zhang, L.: A review of failure modes, condition monitoring and fault diagnosis methods for large-scale wind turbine bearings. Measurement 149, 107002 (2020). doi:10.1016/j.measurement.2019.107002

3. Wang, T., Han, Q., Chu, F., Feng, Z.: Vibration based condition monitoring and fault diagnosis of wind turbine planetary gearbox: A review. Mech Syst Signal Process 126, 662-685 (2019). doi:10.1016/j.ymssp.2019.02.051

4. Yang, J., Huang, D., Zhou, D., Liu, H.: Optimal IMF selection and unknown fault feature extraction for rolling bearings with different defect modes. Measurement 157, 107660 (2020). doi:10.1016/j.measurement.2020.107660

5. Liang, P., Deng, C., Wu, J., Yang, Z.: Intelligent fault diagnosis of rotating machinery via wavelet transform, generative adversarial nets and convolutional neural network. Measurement 159, 107768 (2020). doi:10.1016/j.measurement.2020.107768

6. Zair, M., Rahmoune, C., Benazzouz, D.: Multi-fault diagnosis of rolling bearing using fuzzy entropy of empirical mode decomposition, principal component analysis, and SOM neural network. Proceedings of the Institution of Mechanical Engineers, Part C 233(9), 3317-3328 (2019). doi:10.1177/0954406218805510

7. Chen, B., Shen, B., Chen, F., Tian, H., Xiao, W., Zhang, F., Zhao, C.: Fault diagnosis method based on integration of RSSD and wavelet transform to rolling bearing. Measurement 131, 400411 (2019). doi:10.1016/j.measurement.2018.07.043

8. Cheng, Y., Wang, Z., Chen, B., Zhang, W., Huang, G.: An improved complementary ensemble empirical mode decomposition with adaptive noise and its application to rolling element bearing fault diagnosis. ISA T 91, 218-234 (2019). doi:10.1016/j.isatra.2019.01.038

9. Huang, N.E., Shen, Z., Long, S.R., Wu, M.C., Shih, H.H., Zheng, Q., Yen, N.-C., Tung, C.C., Liu, H.H.: The empirical mode decomposition and the Hilbert spectrum for nonlinear and nonstationary time series analysis. P Roy Soc Lond A Mat 454(1971), 903-995 (1998). doi:10.1098/rspa.1998.0193

10. Wu, Z., Huang, N.E.: Ensemble empirical mode decomposition: a noise-assisted data analysis method. Adv Adapt Data Anal 1(1), 1-41 (2009). doi:10.1142/S1793536909000047

11. Smith, J.S.: The local mean decomposition and its application to EEG perception data. J R Soc Interface 2(5), 443-454 (2005). doi:10.1098/rsif.2005.0058

12. Frei, M.G., Osorio, I.: Intrinsic time-scale decomposition: time-frequency-energy analysis and real-time filtering of non-stationary signals. P R Soc A 463(2078), 321-342 (2007). doi:10.1098/rspa.2006.1761

13. Medina, R., Macancela, J.-C., Lucero, P., Cabrera, D., Cerrada, M., Sánchez, R.-V., Vásquez, R.E.: Vibration signal analysis using symbolic dynamics for gearbox fault diagnosis. Int J Adv Manuf Tech 104(5), 2195-2214 (2019). doi:10.1007/s00170-019-03858-0

14. Lin, J., Chen, Q.: A novel method for feature extraction using crossover characteristics of 
nonlinear data and its application to fault diagnosis of rotary machinery. Mech Syst Signal Process 48(1), 174-187 (2014). doi:10.1016/j.ymssp.2014.04.007

15. Lin, J., Dou, C.: A novel method for condition monitoring of rotating machinery based on statistical linguistic analysis and weighted similarity measures. J Sound Vib 390, 272-288 (2017). doi:10.1016/j.jsv.2016.12.005

16. Lin, J., Chen, Q.: Fault Diagnosis of Rolling Bearings Based on Multifractal Detrended Fluctuation Analysis and Mahalanobis Distance Criterion. Mech Syst Signal Process 38(2), 515 533 (2013). doi:10.1016/j.ymssp.2012.12.014

17. Du, W., Kang, M., Pecht, M.: Fault Diagnosis Using Adaptive Multifractal Detrended Fluctuation Analysis. IEEE T Ind Electron 67(3), 2272-2282 (2019). doi:10.1109/TIE.2019.2892667

18. Gao, Z., Jin, N.: Complex network from time series based on phase space reconstruction. Chaos 19(3), 033137 (2009). doi:10.1063/1.3227736

19. Peng, Y., Xiang, W.: Short-term traffic volume prediction using GA-BP based on wavelet denoising and phase space reconstruction. Physica A 549, 123913 (2020). doi:10.1016/j.physa.2019.123913

20. Kennel, M.B., Brown, R., Abarbanel, H.D.: Determining embedding dimension for phasespace reconstruction using a geometrical construction. Phys Rev A 45(6), 3403 (1992). doi:10.1103/PhysRevA.45.3403

21. Zhang, S., Yang, J., Zhang, J., Liu, H., Hu, E.: On bearing fault diagnosis by nonlinear system resonance. Nonlinear Dynam 98(3), 2035-2052 (2019). doi:10.1007/s11071-019-05305-x

22. Hao, B.L.: Symbolic dynamics and characterization of complexity. Physica D 51(1-3), 161176 (1991). doi:10.1016/0167-2789(91)90229-3

23. Bandt, C., Pompe, B.: Permutation entropy: a natural complexity measure for time series. Phys Rev Lett 88(17), 174102 (2002). doi:10.1103/PhysRevLett.88.174102

24. Yan, R., Liu, Y., Gao, R.X.: Permutation entropy: A nonlinear statistical measure for status characterization of rotary machines. Mech Syst Signal Process 29, 474-484 (2012). doi:10.1016/j.ymssp.2011.11.022

25. Zheng, J., Dong, Z., Pan, H., Ni, Q., Liu, T., Zhang, J.: Composite multi-scale weighted permutation entropy and extreme learning machine based intelligent fault diagnosis for rolling bearing. Measurement 143, 69-80 (2019). doi:10.1016/j.measurement.2019.05.002

26. Tian, Z., Li, S., Wang, Y.: A prediction approach using ensemble empirical mode decomposition-permutation entropy and regularized extreme learning machine for short-term wind speed. Wind Energy 23(2), 177-206 (2020). doi:10.1002/we.2422

27. Landauskas, M., Cao, M., Ragulskis, M.: Permutation entropy-based 2D feature extraction for bearing fault diagnosis. Nonlinear Dynam 102(3), 1717-1731 (2020). doi:10.1007/s11071-02006014-6

28. Aziz, W., Arif, M.: Multiscale permutation entropy of physiological time series. In: 2005 Pakistan Section Multitopic Conference, Karachi, Pakistan, 24-25 Dec., 2005, pp. 1-6. IEEE

29. Parlitz, U., Berg, S., Luther, S., Schirdewan, A., Kurths, J., Wessel, N.: Classifying cardiac biosignals using ordinal pattern statistics and symbolic dynamics. Comput Biol Med 42(3), 319327 (2012). doi:10.1016/j.compbiomed.2011.03.017

30. Liu, B., Peng, J.: Nonlinear Dynamics. Higher Education Press, Beijing (2004) 